\title{
VARIAÇÕES DA ANATOMIA DA CAVIDADE NASAL E DOS SEIOS PARANASAIS - RELATO DE CASO
}

\section{VARIATIONS OF THE NASAL CAVITY AND PARANASAL SINUSES ANATOMY - CASE REPORT}

\author{
Maria José Albuquerque Pereira de Sousa e Tucunduva \\ Jacqueline Dias Bolzan** \\ Thásia Luiz Dias Ferreira* \\ Marina Gazano Baladi*** \\ Cláudio Fróes de Freitas*
}

\section{RESUMO}

A região da cavidade nasal e dos seios paranasais pode apresentar um grande número de variações anatômicas, incluindo alterações das estruturas ósseas que a constituem, pneumatização dessas estruturas ou ainda diversidade no grau de aeração das cavidades já existentes. Essas possibilidades morfológicas podem ocorrer de forma isolada ou em conjunto. Algumas dessas variações, como a pneumatização da concha nasal média, são bastante comuns; já outras, como o seio etmomaxilar, raramente são observadas. Neste trabalho é relatado um caso no qual foram encontradas diferentes variações em um mesmo paciente, sem que houvesse alteração da mucosa, sugestivo de afecção.

DESCRITORES: Cavidade nasal, Seios paranasais, tomografia computadorizada, imagem

\section{ABSTRACT}

The nasal cavity and paranasal sinuses region can present a large number of variations, including changes in bony structures that constitute pneumatization or even diversity of these structures due to the degree of aeration. These morphological possibilities may occur isolated or together. Some of these variations, as pneumatization of the middle nasal concha, are quite common, while others, like the etmomaxilar sinus are rarely observed. We report a case in which different variations were found in the same patient, with no mucosal abnormality that would suggest the presence of disease.

DESCRIPTORS: Nasal cavity, Paranasal sinuses, Computed tomography, Image

\footnotetext{
*** Prof Dr da Universidade de São Paulo e da Universidade Cidade de São Paulo, maria.tucunduva@unicid.edu.br

** Biomédica formada pela Universidade Cidade de São Paulo

*** Prof Ms da Universidade de São Paulo
} 
TUCUNDUVA MJAPS BOLZAN JD

FERREIRA TLD

BALADI MG

FREITAS CF

VARIAÇÕES DA

ANATOMIA DA

CAVIDADE NASAL

E DOS SEIOS

PARANASAIS

RELATO DE CASO

84

REV, ODONTOL

UNIV, CID, SÃO

PAULO

2013; 25(1): 83-7,

JAN-ABR
REVISÃO DA L I TERATURA

Os seios paranasais são cavidades duplas, sendo dois frontais, dois maxilares, dois esfenoidais e dois grupos de células etmoidais. Os seios frontais apresentam uma variedade morfológica grande o suficiente para que tenham sido utilizados como recurso forense de identificação individual ${ }^{1}$. Essa classificação apresentou como critérios a área, a forma e a simetria. O seio maxilar foi descrito por Kayalioglu et al. ${ }^{2}$ (2000) mostrando septos, duplicação, podendo estar hipoplásico ou, ainda, haver agenesia dessas cavidades. A possibilidade de essa cavidade ter seus limites além daqueles da órbita, indicaria a sua hiperplasia, de acordo com Sirikçi et al. ${ }^{3}$ (2000). As células etmoidais foram descritas como um conjunto de células cujas expansões ocorreriam em várias direções, provocando o compartilhamento do espaço entre as diversas estruturas da cavidade nasal e dos seios paranasais ${ }^{4,5,6}$ descreveram a pneumatização do teto da órbita pela presença de células etmoidais anteriores denominadas supraorbitais. Esses mesmos autores discorreram sobre a presença de células etmoidais no soalho da órbita, as células infraorbitais (Haller). Khanobthamchai et al. ${ }^{7}$ (1990) relataram a presença de células etmoidais posteriores que disputariam pelo espaço do seio maxilar, e que seriam identificadas pela drenagem do seu espaço aéreo no meato

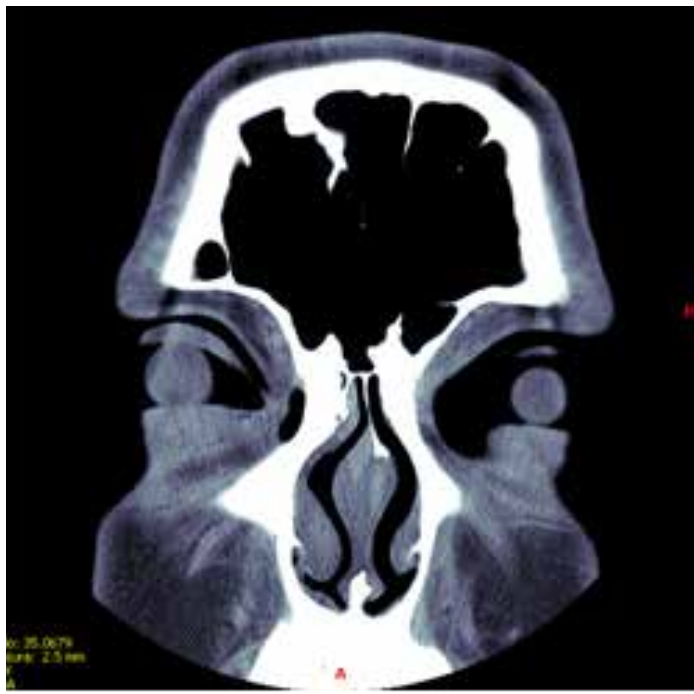

Figura 1 - Hiperplasia do seio frontal superior, ao passo que a drenagem do seio maxilar ocorreria sempre no meato médio. Ainda Jorissen et al.8 (1997), Kayalioglu et al. $^{2}$ (2000) e Bradley e Kountakis ${ }^{9}$ (2004) chamaram a atenção para a célula da crista do nariz (agger nasi), localizada entre o ducto lácrimonasal e a bolha etmoidal e a célula esfeno-etmoidal (Onodi), que compartilharia o espaço com o seio esfenoidal.

No tocante às alterações ósseas da cavidade nasal, Ferrie et al. ${ }^{4}$ (1991), Meloni et al. ${ }^{5}$ (1992) e Ünlü et al. ${ }^{6}$ (1994) relataram a possibilidade da concha nasal média encontrar-se pneumatizada, quando uma aeração separava as corticais medial e lateral da concha ou com curvatura paradoxal, ou seja, com a concavidade voltada para o septo nasal. O desvio de septo, associado ou não a alterações das conchas nasais, foi relatado por Uygur et al. ${ }^{11}$ (2003) como a variação mais comum da cavidade nasal.

Neste trabalho, apresentamos um grande número dessas variações em um mesmo indivíduo.

\section{MÉTODOS}

Um paciente, gênero feminino, 19 anos de idade cronológica, apresentou-se para exame de tomografia computadorizada helicoidal, para investigação de sinusite. O exame constou de cortes coronais, realizados a partir da glabela até

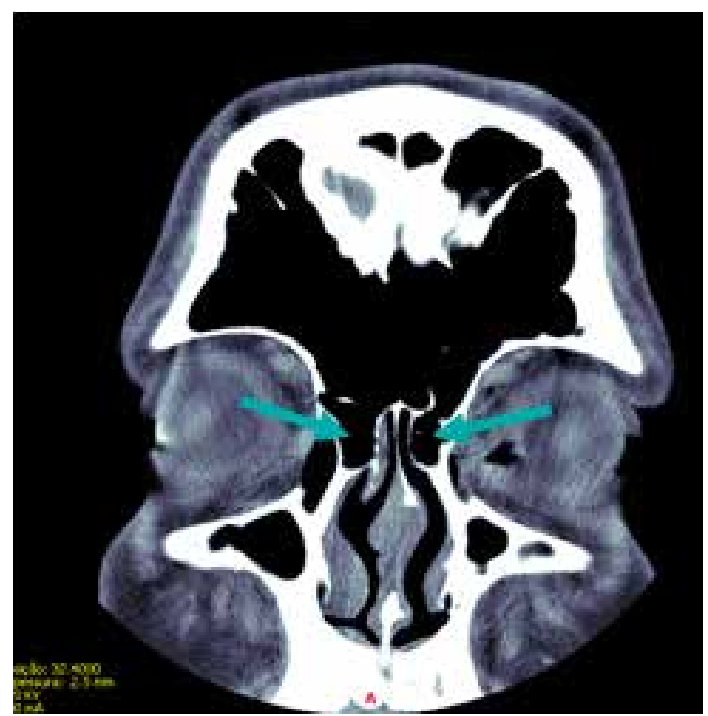

Figura 2 - Célula da crista do nariz com célula anexial à esquerda bilateralmente 


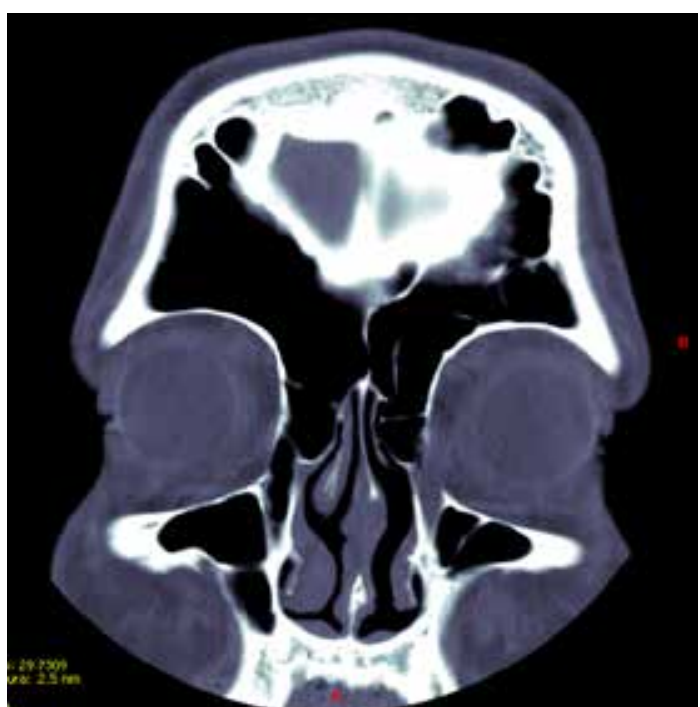

Figura 3 - Septação do seio maxilar

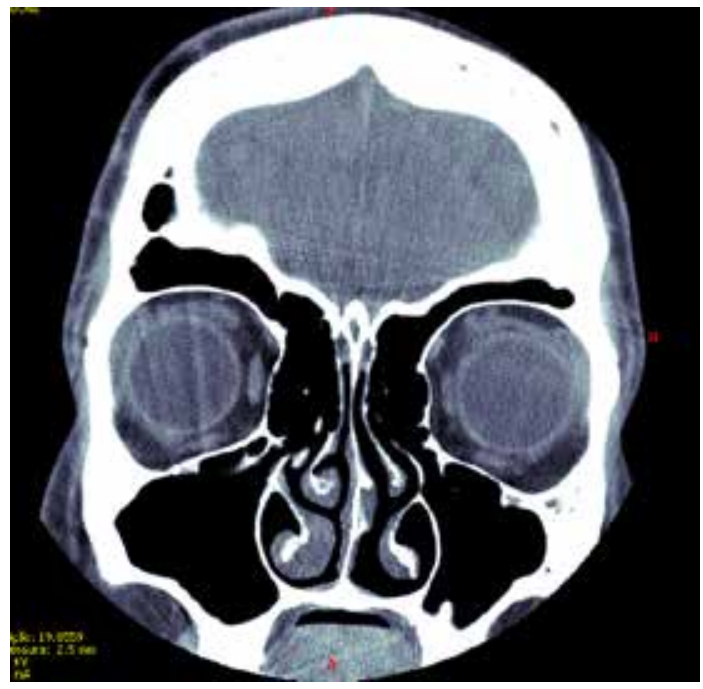

Figura 5 - Célula infraorbital

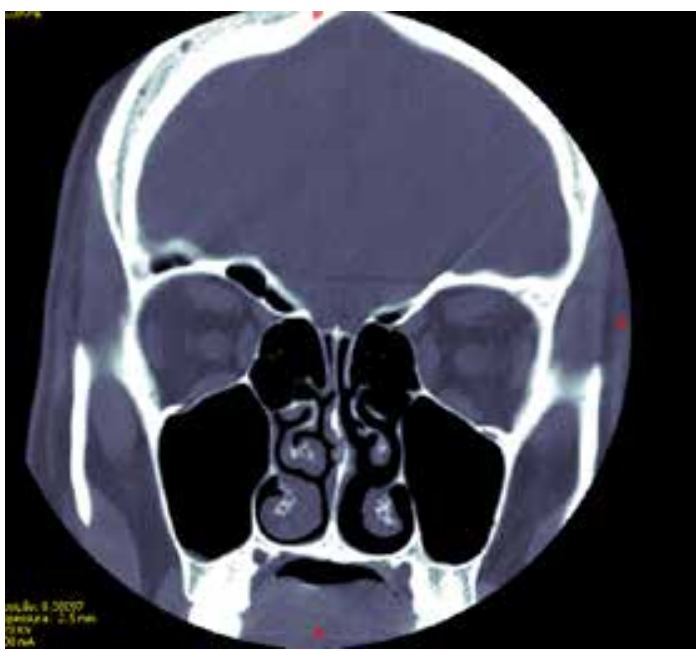

Figura 7 - Pneumatização do processo

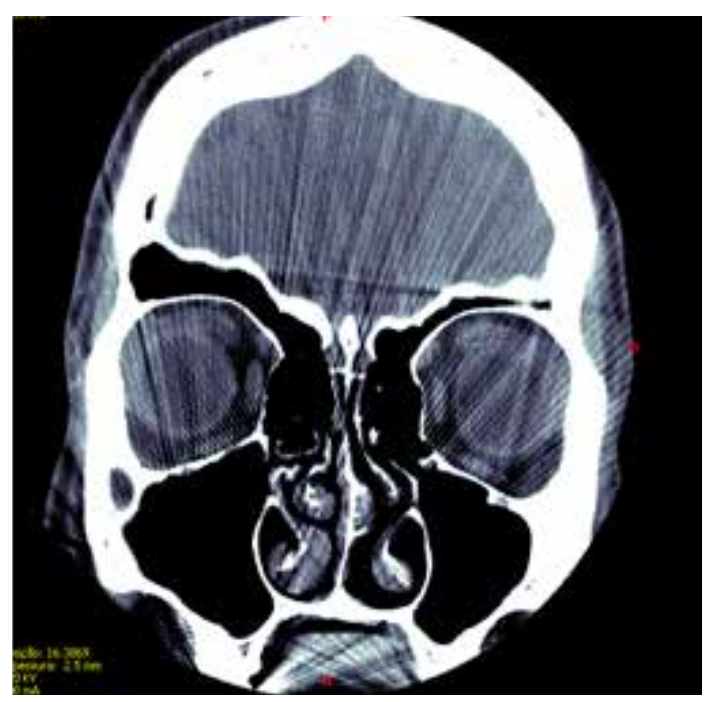

Figura 4 - Hiperplasia do seio maxilar

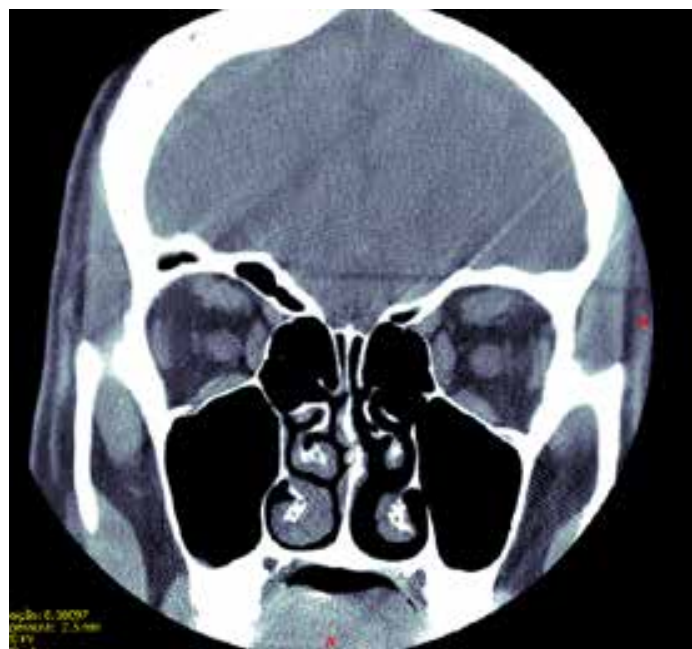

Figura 6 - Célula supraorbital bilateralmente bilateralmente

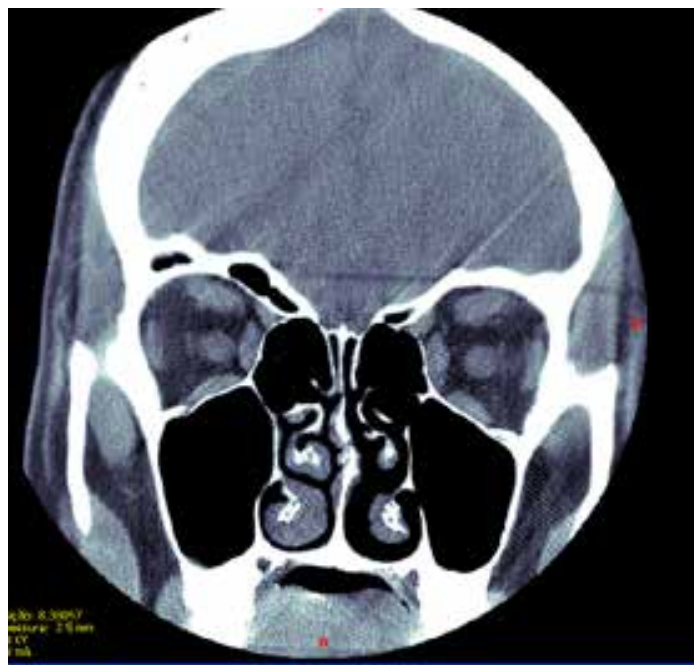

Figura 8 - Curvatura paradoxal da concha uncinado à direita nasal média
TUCUNDUVA MJAPS

BOLZAN JD

FERREIRA TLD

BALADI MG

FREITAS CF

VARIAÇÕES DA

ANATOMIA DA

CAVIDADE NASAL

E DOS SEIOS

PARANASAIS

RELATO DE CASO

\section{5}

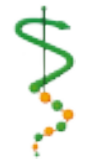

REV, ODONTOL. UNIV, CID, SÃo PAULO

2013; 25(1): $83-7$ JAN - ABR 


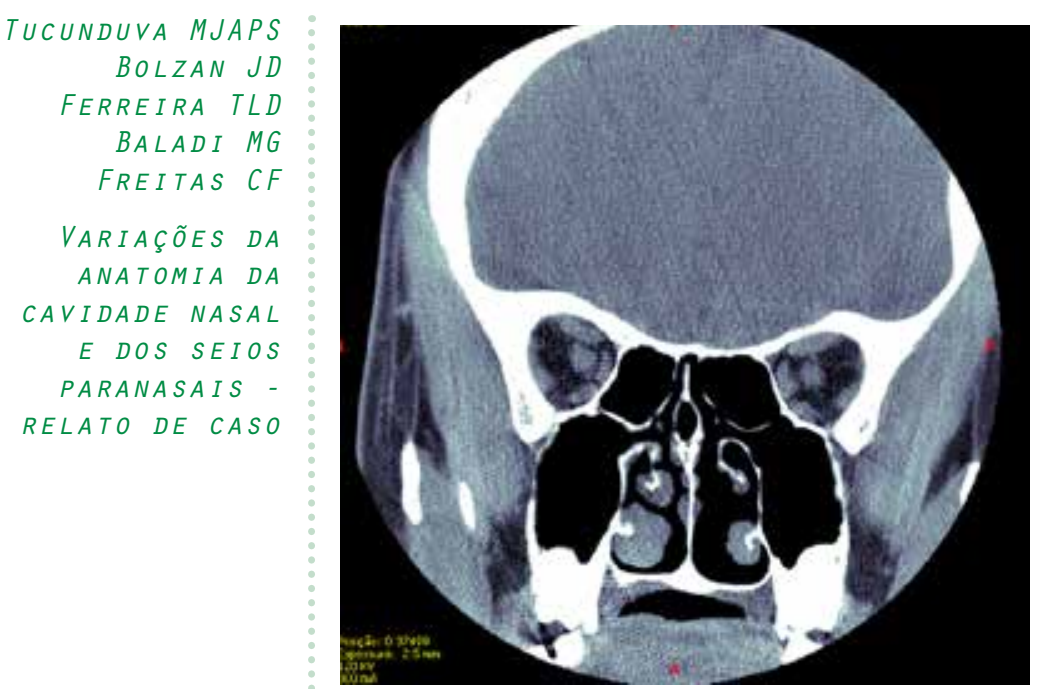

Figura 9 - Pneumatização do septo nasal

o dorso da sela turca e axiais, da calota craniana até os arcos dentais superiores, com 2,5mm de espessura, com regime de $120 \mathrm{kv}, 330 \mathrm{~mA}$.

\section{RESULTADOS}

Foi observada hiperplasia do seio frontal, com célula anexial à esquerda e extensão horizontal, para o teto da órbita, célula da crista do nariz bilateralmente, 86 célula supraorbital bilateralmente, célula infraorbital bilateralmente, curvatura paradoxal da concha nasal média bilateralmente, septações e hiperplasia do seio maxilar, seio etmomaxilar bilateralmente, pneumatização do processo uncinado bilateralmente, pneumatização da porção basal da concha média à $\mathrm{D}$ e pneumatização do septo nasal. O seio esfenoidal apresentou características normais.

\section{I SCUSSÃO}

O seio maxilar foi descrito por Kayalioglu et al. ${ }^{2}$ (2000) mostrando septações e apresentando condições de hipoplasia e agenesia. A possibilidade de que essa cavidade apresente os seus limites para além dos da órbita indicaria a hiperplasia desse seio, de acordo com Sirikçi et al. (2000). No presente estudo, a paciente apresentou septos no seio maxilar e hiperplasia dos dois lados.

Ferrie et al. ${ }^{4}$ (1991) e Meloni et al. ${ }^{5}$ (1992) descreveram a pneumatização do teto da órbita pela presença de células etmoidais anteriores chamadas supraorbitais. Estes autores referiram-se à presença

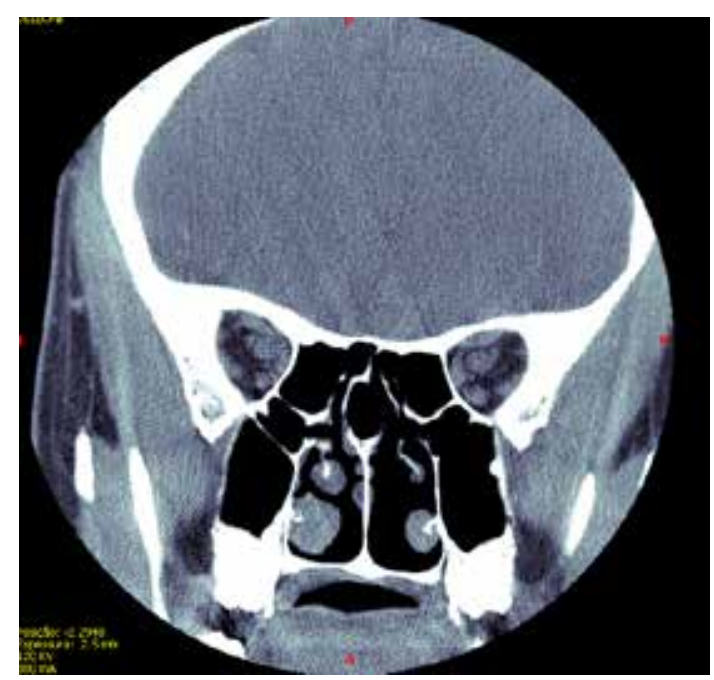

Figura 10 - Seio etmomaxilar bilateralmente

de células etmoidais no soalho da órbita para as células de infraorbital. Ambos os tipos de células, as células infraorbitais e supraorbitais, foram observados bilateralmente nas imagens da paciente apresentada neste trabalho. Khanobthamchai et al. ${ }^{7}$ (1991) relataram a presença de células etmoidais posteriores que iriam disputar pelo espaço dos seios maxilares, e seriam identificadas pela drenagem no meato superior, enquanto a drenagem dos seios maxilares sempre aconteceria no meato médio. Jorissen et al. ${ }^{8}$ (1997) chamaram a atenção para a célula da crista do nariz (agger nasi), localizada entre o ducto lacrimonasal e a célula etmoidal e a célula esfenoetmoidal, que divide espaço com o seio esfenoidal.

Em relação às alterações ósseas da cavidade nasal, Bolger et al. ${ }^{12}$ (1991) relataram a possibilidade de pneumatização da concha nasal média, encontrada quando ocorre uma aeração entre as corticais medial e lateral da concha ou ainda a presença de curvatura paradoxal, ou seja, a concavidade da concha voltada para o septo nasal. Tais situações também foram encontradas na paciente aqui descrita.

O desvio do septo, com ou sem alterações das conchas nasais associadas foi relacionado por Uygur et al. ${ }^{11}$ (2003) como a variação mais comum da cavidade nasal, que também esteve presente nas imagens aqui relatadas. Embora neste exame pudessem ser observadas em grande número as variações das estruturas constituintes da cavidade nasal e dos seios para- 
nasais, não foi observado o espessamento da mucosa que caracteriza a presença de afecção.

\section{CONCLUSÃO}

Embora neste caso a mucosa não tenha apresentado espessamento sugestivo de afecção, as variações anatômicas que acometem a cavidade nasal e os seios paranasais podem predispor o indivíduo a tal situação e devem, portanto, ser observadas pelo radiologista. Essas variações podem ocorrer isoladamente ou em conjunto, em uma mesma pessoa.

\section{REFERÊNCIAS}

1. Yoshino M, Miyasaka S, Sato H, Seta S. Classification system of frontal sinus patterns by radiography. Its application to identification of unknown skeletal remains. Forensic Sci Int 1987 Aug;34(4):289-99.

2. Kayalioglu G, Oyar O, Govsa F. Nasal cavity and paranasal sinus bony variations: a computed tomographic study. Rhinology 2000 Sep;38(3):108-13.

3. Sirikci A, Bayazit Y, Gumusburun E, Bayram M, Kanlikana M. A new approach to the classification of maxillary sinus hypoplasia with relevant clinical implications. Surg Radiol Anat 2000 22(5-6):243-7.

4. Ferrie JC, Azais O, Vandermarcq P, Klossek JM, Drouineau J, Gasquet C. [X-ray computed tomographic study of the ethmoid and middle meatus. II. Radio-anatomy (axial incidence) and morphological variations]. J Radio/ 1991 Oct;72(10):477-87.

5. Meloni F MR, Rovasio S, Stomeo F, Teatini GP. Anatomic variations of surgical importance in ethmoid labyrinth and sphenoid sinus. a study of radiological anatomy. Surg Radiol Anat 1992 14(65-70.

6. Arslan H, Aydinlioglu A, Bozkurt M, Egeli E. Anatomic variations of the paranasal sinuses: CT examination for endoscopic sinus surgery. Auris Nasus Larynx 1999 Jan;26(1):39-48.

7. Khanobthamchai K, Shankar L, Hawke M, Bingham B. Ethmomaxillary sinus and hypoplasia of maxillary sinus. J Otolaryngol 1991 Dec;20(6):425-7.

8. Jorissen M, Hermans R, Bertrand B, Eloy P. Anatomical variations and sinusitis. Acta Otorhinolaryngol Belg 1997 51(4):219-26.

9. Bradley DT, Kountakis SE. The role of agger nasi air cells in patients requiring revision endoscopic frontal sinus surgery. Otolaryngol Head Neck Surg 2004 Oct;131(4):525-7.

10. Unlu HH, Akyar S, Caylan R, Nalca Y. Concha bullosa. J Otolaryngol 1994 Feb;23(1):23-7.

11. Uygur K, Tuz M, Dogru H. The correlation between septal deviation and concha bullosa. Otolaryngol Head Neck Surg 2003 Jul;129(1):33-6.

12. Bolger WE, Butzin CA, Parsons DS. Paranasal sinus bony anatomic variations and mucosal abnormalities: CT analysis for endoscopic sinus surgery. Laryngoscope 1991 Jan;101(1 Pt 1):56-64.

Recebido em: 20/12/2012

Aceito em: 07/01/2013 